\section{Integrative Medicine \\ International}

\title{
China's Tu Youyou, Nobel Laureate in Medicine 2015, and Relationships with the TCM Research Center Graz at the Medical University
}

\author{
Daniela Litscher Gerhard Litscher \\ Research Unit for Complementary and Integrative Laser Medicine, Research Unit of Biomedical \\ Engineering in Anesthesia and Intensive Care Medicine, and TCM Research Center Graz, Medical \\ University of Graz, Graz, Austria
}

Key Words
Malaria · Qinghao $\cdot$ Sweet wormwood $\cdot$ Artemisia $\cdot$ Moxibustion · Acupuncture

\section{Abstract}

For the first time in history, the Nobel Prize in Medicine 2015 was awarded to a researcher who is specialized in traditional Chinese medicine. The Chinese Tu Youyou received this highest award in medicine for her excellent work in malaria research. Tu Youyou and her team found out that artemisinin, the active ingredient of the medicinal herb 'sweet wormwood', represents an effective malaria therapy. In the following article, the research that led to this Nobel Prize is introduced. In addition, the insidious tropical disease malaria will be explained briefly. Results of the research on moxibustion using Artemisia are also presented in this article; these studies were carried out at the TCM Research Center Graz, partly in close cooperation with the China Academy of Chinese Medical Sciences, the institution of the Nobel Prize winner.

\section{Introduction}

This year's Nobel Prize in Medicine was awarded to 3 scientists on October 5, 2015. Satoshi Omura and William C. Campbell received one half of the prize for their research on a therapy against infections caused by roundworm parasites. The second half of this renowned prize, and this is truly a sensation, was awarded for the first time in history to a researcher who specializes 
Integrative

International
Integr Med Int 2016;3:1-9

DOI: $10.1159 / 000442817$

Litscher and Litscher: China's Tu Youyou, Nobel Laureate in Medicine 2015, and Relationships with the TCM Research Center Graz at the Medical University
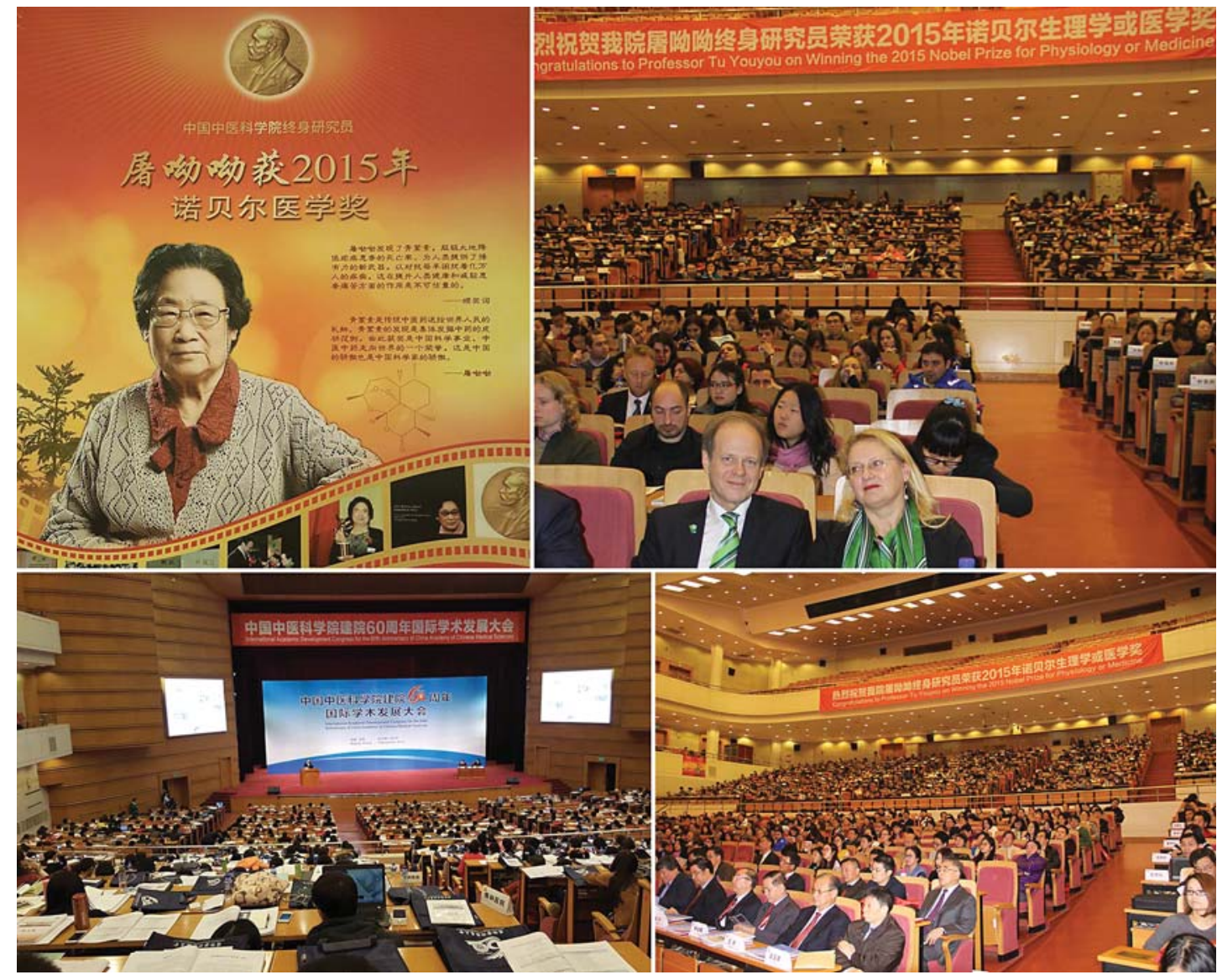

Fig. 1. Tu Youyou (top left). The vice president of the Medical University of Graz, Prof. Irmgard Lippe, and the head of the TCM Research Center Graz, Prof. Gerhard Litscher (both top right), had the opportunity to convey congratulations on the occasion of the International Academic Development Congress for the 60th anniversary of the China Academy of Chinese Medical Sciences on November 7, 2015.

in traditional Chinese medicine (TCM). The Chinese Tu Youyou (fig. 1) from the China Academy of Chinese Medical Sciences was awarded the Nobel Prize for Medicine and Physiology, the highest award for physicians, for her excellent work on sweet wormwood (Artemisia annua) in malaria research [1]. In the present article, the Chinese Nobel Prize winner will be presented, and the disease malaria will be described in greater detail. Our research team at the TCM Research Center at the Medical University of Graz has been working with numerous scientists from the China Academy of Chinese Medical Sciences for many years. For this reason, joint research results of both institutions on topics related to that of the Nobel Prize winner are presented in this work.

\section{Tu Youyou Is Awarded the First Nobel Prize in Medicine for TCM}

The Nobel Prize winner Tu Youyou was born in 1930 in Ningbo, a city in eastern China. She studied pharmacy at the Beijing Medical College, today's renowned Peking University Health Science Center. After her excellent graduation, she began working at the Institute of Materia Medica of the China Academy of Chinese Medical Sciences, where she spent her entire academic career (fig. 2). 
Integrative

Medicine

2

\begin{tabular}{l|l}
\hline Integr Med Int 2016;3:1-9 \\
\hline DOI: 10.1159/000442817 & $\begin{array}{l}\text { @ 2015 The Author(s). Published by S. Karger AG, Basel } \\
\text { www.karger.com/imi }\end{array}$ \\
\hline
\end{tabular}

Litscher and Litscher: China's Tu Youyou, Nobel Laureate in Medicine 2015, and Relationships with the TCM Research Center Graz at the Medical University

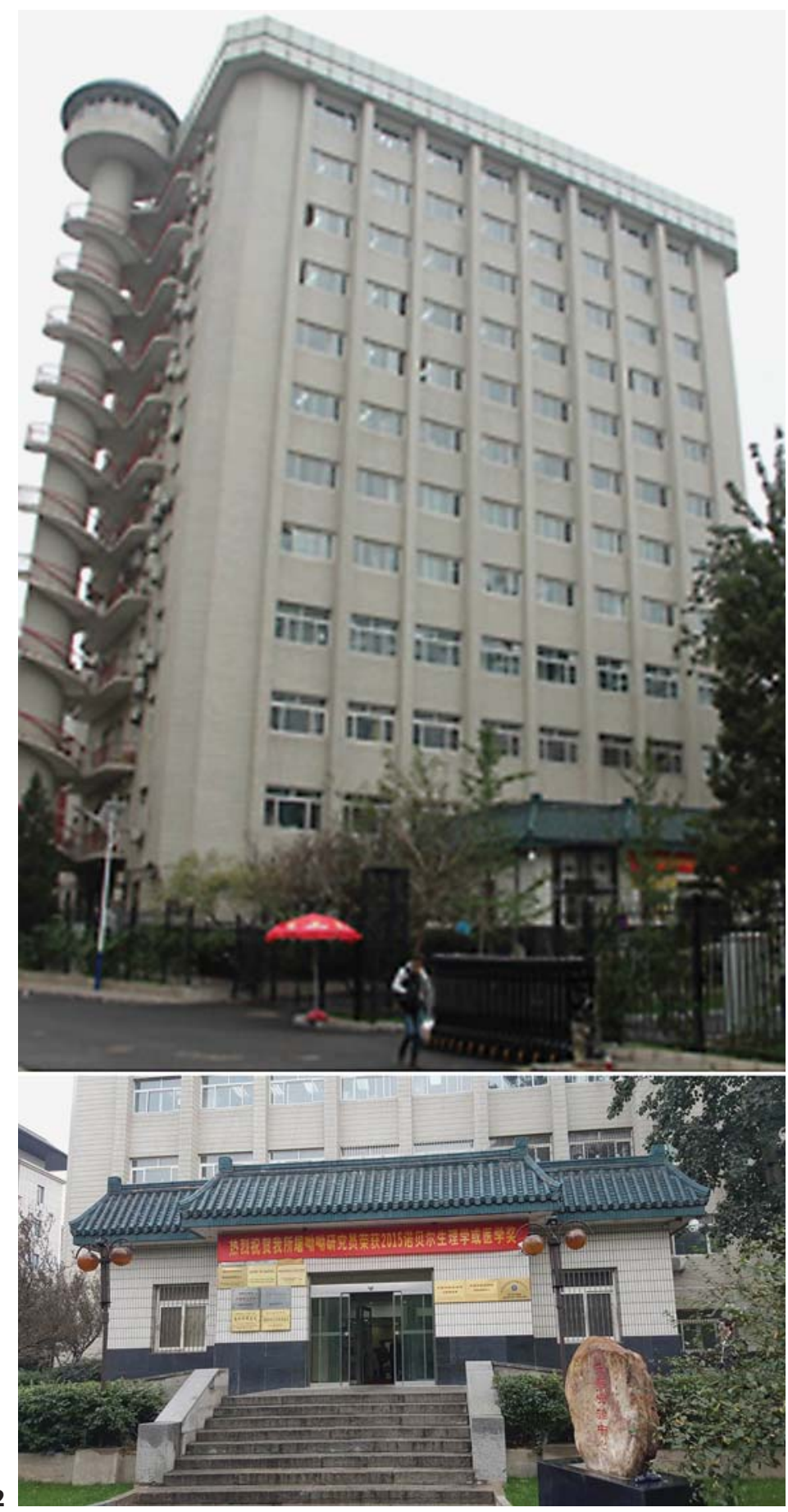

(For legend see next page.) 


\section{Integrative \\ Medicine}

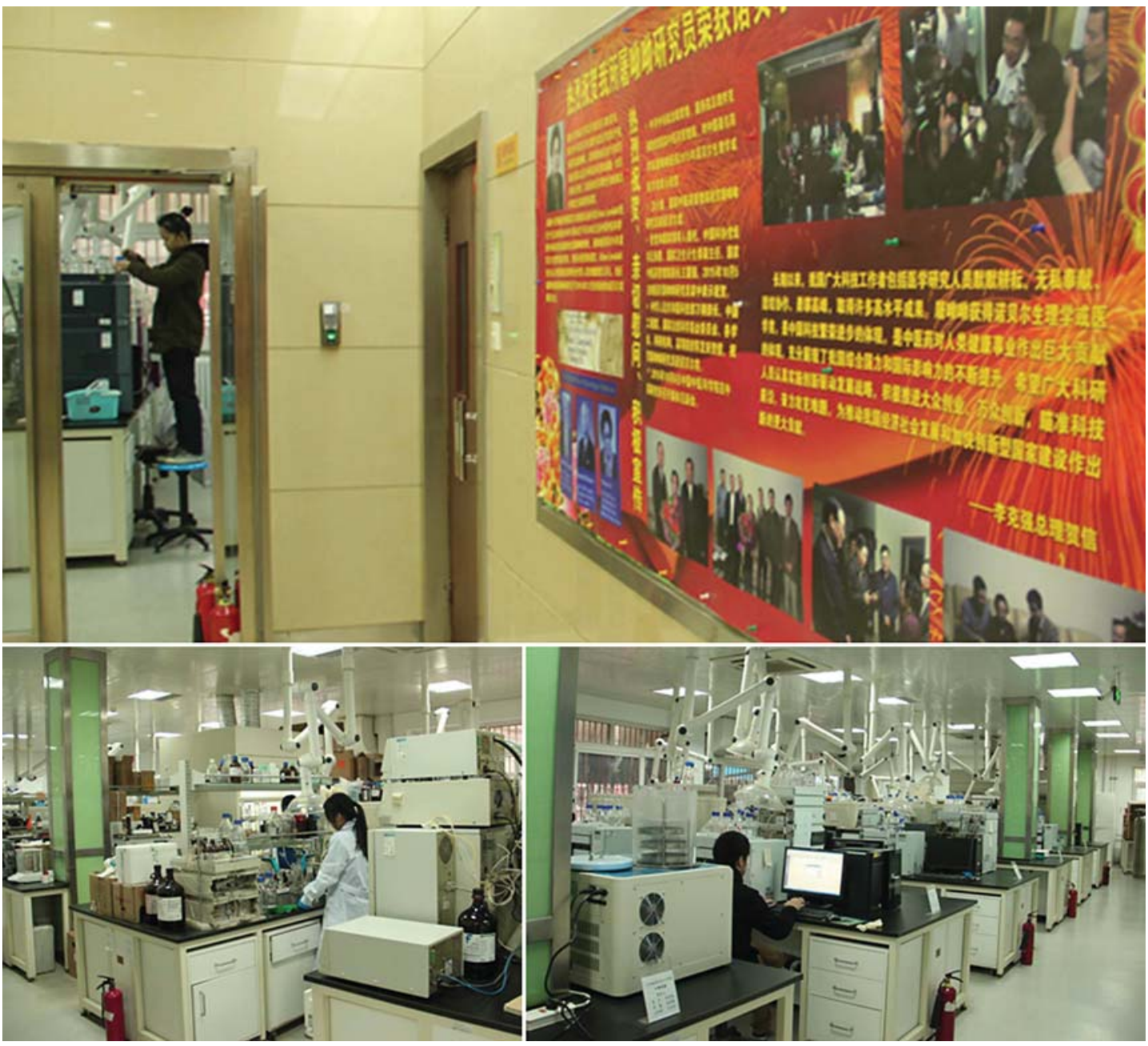

Fig. 2. Workplace and office of the Nobel Laureate Tu Youyou at the China Academy of Chinese Medical Sciences. The photos were taken during a cooperation visit of Prof. Gerhard Litscher in Beijing on October 21, 2015.

recipes of more than 640 medicinal herbs. Tu then reduced this selection to a few. Qinghao (the Chinese name for A. annua) turned out to be one of the most promising plants.

Sweet wormwood has been used for medical purposes for a long time. The oldest document proving the medical use of qinghao dates back to 168 BCE. On a piece of silk, the use of qinghao for treating hemorrhoids is described. Another document from the Ming dynasty reports the effectiveness of qinghao against fever.

After several trials with the medicinal herb and additional literature research, Tu Youyou found the decisive clue in the Handbook of Prescriptions for Emergencies, written by the physicist Ge Hong (284-363). The following recipe can be found in this book: 'A bunch of qinghao is mixed with 2.2 liters of water. Then the juice is pressed out and administered orally to the patient'. Thus, Tu found out that the active ingredients of sweet wormwood might be destroyed by the heating process during the extraction, and consequently the extract would not be fully effective. In follow-up experiments, the Nobel Laureate set up experiments using solvents with lower boiling points. The components were separated into acidic and neutral extracts. The neutral extract with the number 191 was to bring about the breakthrough. Qing- 
haosu (the name of the extract) showed an effectiveness of $100 \%$ in parasitemia. The extract showed an excellent effect in Plasmodium berghei-infected mice as well as in monkeys infected with P. cynomolgi. Later on, qinghaosu was called artemisinin.

In the following years, a lot of additional work was done. Among other things, Tu found out that only fresh leaves of sweet wormwood contain the highly effective artemisinin. She also noted that the plant contains the largest amount of artemisinin at the beginning of the flowering period.

To discover any possible side effects, Tu and her team also tested the new drug on themselves. After the successful completion of the experiments, the research team traveled to Hainan in order to verify the research results. Qinghao was tested in 21 patients who were infected with P. vivax and/or P. falciparum. The antipyretic effect occurred very fast. The parasites in the patients' blood decreased significantly compared to a control group. In subsequent experiments, it was shown that artemisinin is able to effectively combat malaria at all stages.

In 1979, the first English publication on the subject was published. At that time, artemisinin had already been successfully tested on more than 2,000 patients. In the following years, the new effective therapy against malaria reached worldwide attention [2]. But it still took some time before skeptics in the western world were convinced of the effectiveness of the herbal ingredient. Only in the 1990s did a western pharmaceutical company bring a combined preparation with artemisinin on the market, which represented another milestone [3].

In 2011, Tu Youyou was awarded the 'Lasker-DeBakey Clinical Medical Research Award', which is of major international importance [2], for her great achievements in research. The corner stone for a successful drug for malaria therapy was therefore set early. The following outstanding research on artemisinin ultimately led to Tu Youyou receiving the Nobel Prize for Medicine and Physiology in 2015 [1].

\section{Malaria: A Brief Description of the Tropical Disease}

The tropical disease malaria is one of the world's most widespread infectious diseases. It occurs in the tropical and subtropical regions of all continents, except Australia. Africa is affected the most, accounting for about $90 \%$ of the cases. In areas where malaria is endemic, an estimated 200 million people are diagnosed with the disease per year. Worldwide, about 600,000 people die from malaria each year [4].

Malaria is caused by parasites of the genus Plasmodium. Plasmodia are intracellular parasites; their development cycle runs in two parts (human host and mosquito vector). The sporozoites, which are introduced during the blood meal of the infected female Anopheles mosquito (mosquito vector), quickly travel from the bloodstream into the liver where they infect hepatocytes and start multiplying. Later on, they differentiate and yield merozoites which escape back into the bloodstream and infect red blood cells. Once these decompose, the merozoites are released and will attack other erythrocytes where some of them differentiate into sexual forms, giving rise to macro- and microgametocytes. These macro- and microgametes are again ingested by the anopheles mosquito; during this stage, they unite and form an oocyst. Finally, the sporozoites emerging from that can infect a new host via saliva (fig. 3) [4].

There are different forms of malaria; the most dangerous type is malaria tropica. Left untreated, it is associated with a mortality rate of up to $20 \%$. Common signs are a general malaise or headache and body aches. In addition, irregular febrile temperature increases can occur with diarrhea. About $60 \%$ of patients develop thrombocytopenia. Cerebral malaria is characterized by convulsions and altered consciousness leading to coma. Furthermore, it may lead to acute renal failure, circulatory collapse, and hemolytic anemia [4]. 


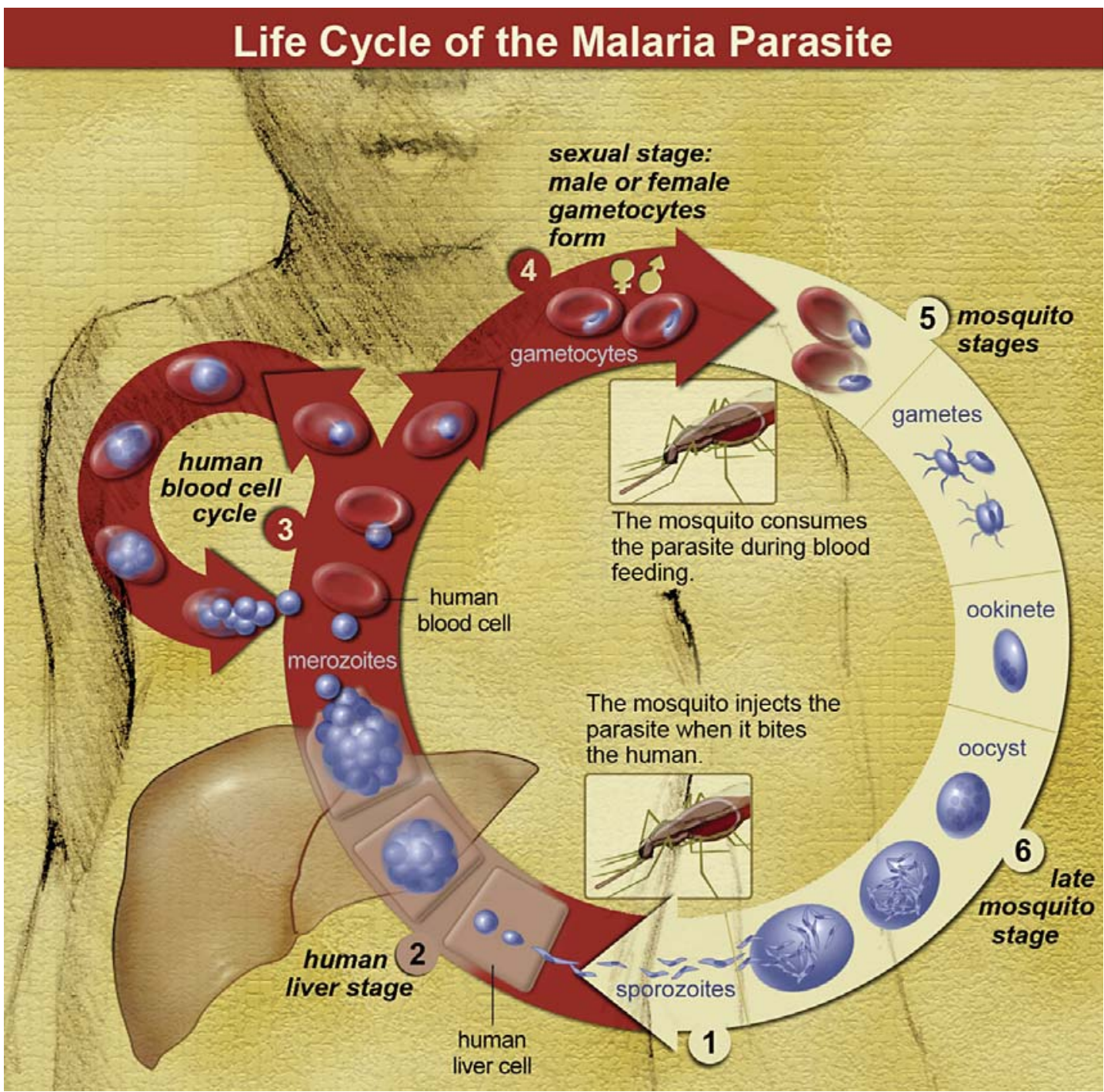

Fig. 3. Cycle of a malaria infection (with permission from: https://upload.wikimedia.org/wikipedia/commons/9/9a/Life_Cycle_of_the_Malaria_Parasite.jpg).

\section{The Medicinal Plant Mugwort in TCM: Acupuncture and Moxibustion}

The outstanding research results that led to the Nobel Prize for Tu Youyou deal with the medicinal plant mugwort. This very special herb, however, is used elsewhere in TCM, too, not 'just' to fight malaria. The mugwort leaves are used for moxibustion (fig. 4). According to traditional methods, mugwort leaves are rolled into small cones or cylinders. These are lit, so they glow and emit heat. The smoldering moxa cones are applied on specific acupuncture points on the skin, where they generate heat and stimulate the acupuncture points. Before the skin gets too hot, the moxa cones are removed [5]. The application via an acupuncture needle including a wooden box is often used in TCM practice in China (fig. 4).

Our research group at the TCM Research Center Graz also performed important research in connection with the herb mugwort and moxibustion. A publication from 2009, which was performed together with the institution at which the Nobel Laureate Tu Youyou works, discusses among other things temperature changes during moxibustion with mugwort. Moxi- 


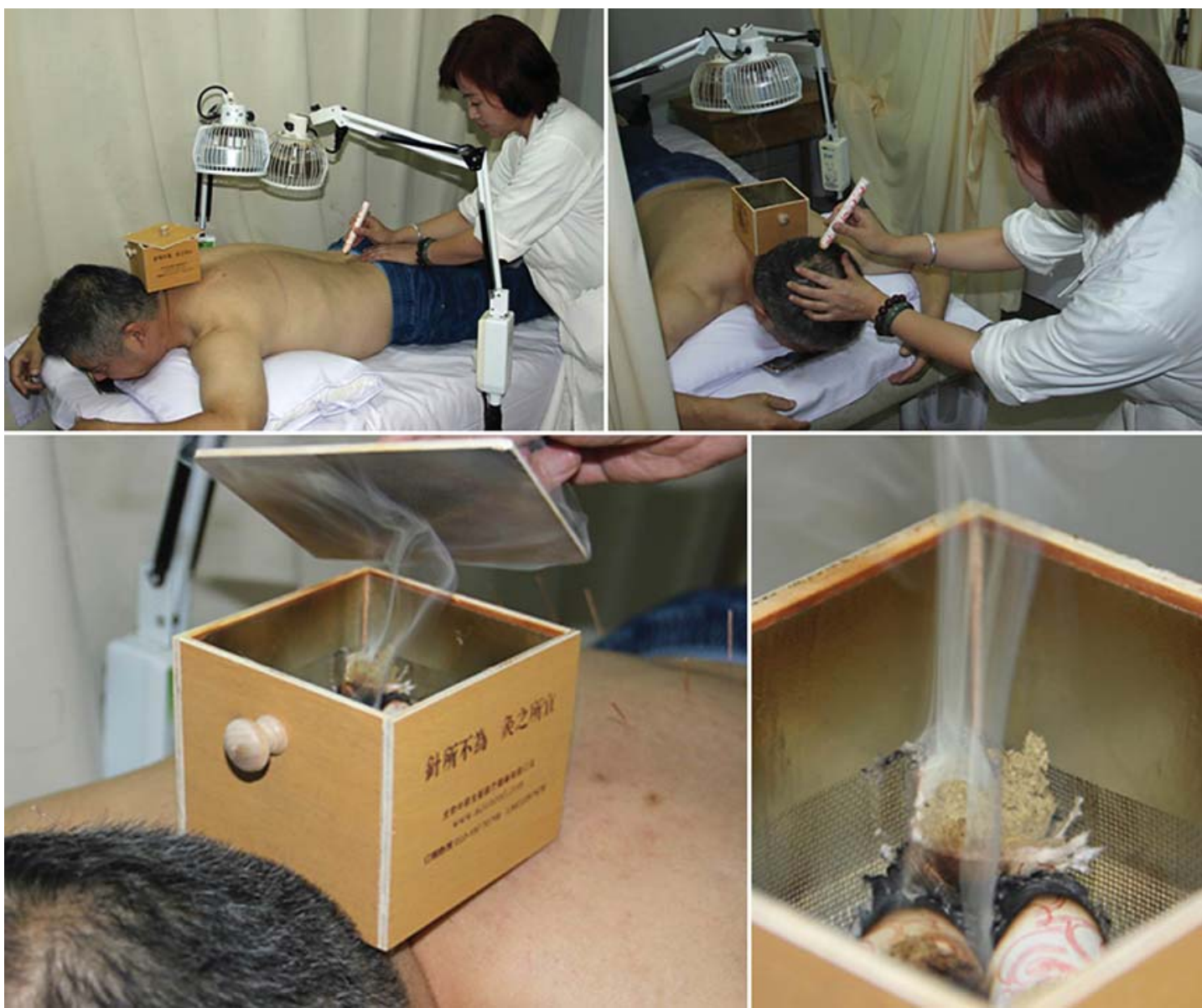

Fig. 4. Moxibustion research at the China Academy of Chinese Medical Sciences in cooperation with the TCM Research Center at the Medical University of Graz, Austria (October 2015).

bustion was performed in healthy volunteers, at point CV 6 (Qihai). In addition to infrared thermography, microcirculation measurements were performed using laser Doppler flowmetry. The results showed positive effects on the treated subjects [6].

Another paper on moxibustion with mugwort, which was written in cooperation with the TCM Research Center Graz, is entitled 'Effects of moxa (A. vulgaris) smoke inhalation on heart rate and its variability' [7]. In this study, 24 healthy volunteers were exposed to moxa smoke from A. vulgaris. Parameters of heart rate variability were measured before, during and after the inhalation. The healthy volunteers showed a significant reduction of the heart rate as well as significant changes in the heart rate variability parameters. The authors came to the conclusion that moxa smoke from the herb mugwort can improve the activity of the autonomic nervous system. The inhaled smoke also had a sedative effect on the human body [7].

In 2010, a scientific review article on the topic moxibustion using mugwort was released. Temperature measurements provided evidence that the increase in surface temperature following moxibustion was not only a short-term effect, but persisted for a longer period of time. The area of the temperature increase on the skin surface could be verified and objectified very well with imaging methods. It should also be mentioned that now also new types of infrared and laser moxa devices are available [8].

A diploma thesis entitled 'Moxibustion - Aktuelle naturwissenschaftliche Betrachtungen' ('Moxibustion - Recent scientific considerations') was performed at the TCM Research Center 

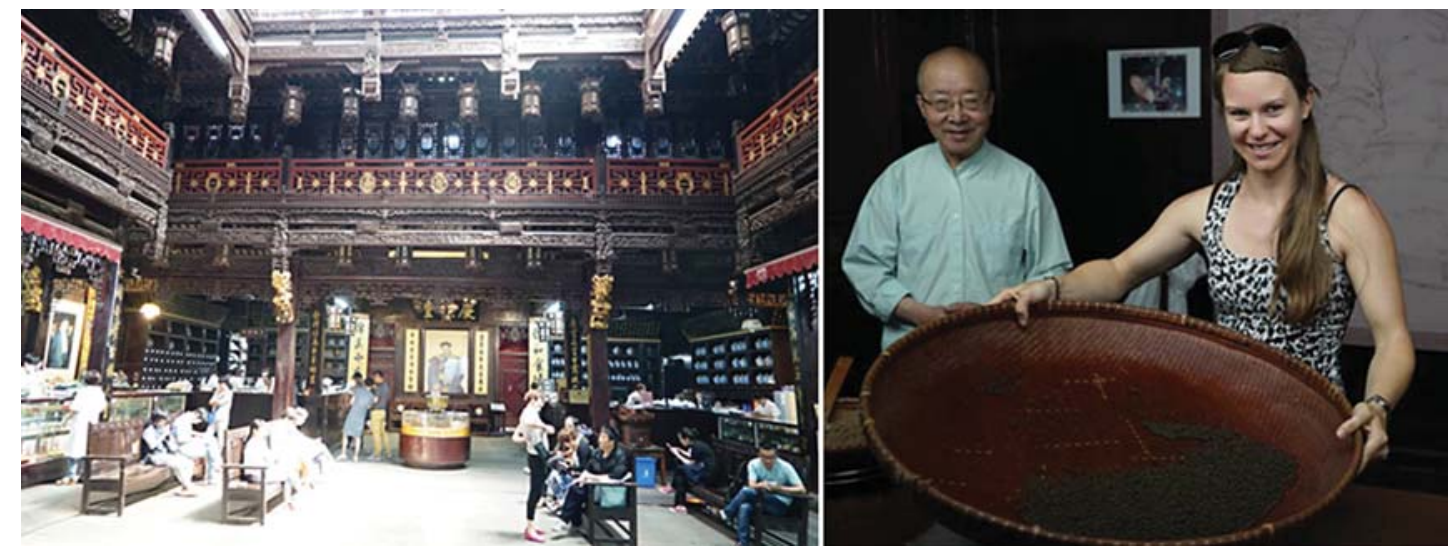

Fig. 5. The oldest TCM pharmacy in China (Daniela Litscher, MSc, PhD; Hangzhou, 2015).

Graz. In cooperation with Chinese researchers, heart rate variability measurements in 20 subjects were performed before, during and after moxibustion at the acupoint CV 4 (Guanyuan). Two moxa methods were compared: needle moxibustion and the so-called moxa stick method (fig. 4). Both in the needle moxa group and in the moxa stick group, the mean heart rate was reduced significantly during the moxa treatment. After the treatment, the heart rate again reached baseline levels. In addition to that, the influence of moxibustion on the autonomic nervous system was examined using different evaluation parameters [9].

All these results show that mugwort, for the investigation of which Tu Youyou was awarded the highest award in science, is not only effective in treating malaria, but can also help treating a lot of other symptoms.

In a recent article, the Chinese newspaper China Daily reported that TCM products are successfully used outside of China (fig. 5). It is stated that TCM products are exported to 154 nations worldwide. Ginseng and goji berries are the most popular products. The famous Tong Ren Tang pharmacy has stores in 15 countries, and it is reported that the export of sweet wormwood already increased following the Nobel Prize being awarded to Tu Youyou [10].

\section{Acknowledgments}

Grateful thanks to the vice president of the China Academy of Chinese Medical Sciences, Prof. Fan Jiping, for the continuous hospitality and the numerous discussions during the SinoAustrian meeting in Beijing in October 2015. The authors also would like to thank Associate Prof. Wang Guangjun who made a personal visit to the working place of Nobel Prize Laureate Prof. Tu Youyou possible. Warm thanks to Prof. Wu Zhongchao for the long-term cooperation in the field of acupuncture and moxa research, and also for permission to use the figures of moxibustion.

Further, the authors would like to thank Prof. Lu Wang and Ingrid Gaischek, MSc, from the Medical University of Graz, for their help in preparing this paper.

\section{Disclosure Statement}

Both authors declare that there is no conflict of interest in connection with the publication of this paper. 
Litscher and Litscher: China's Tu Youyou, Nobel Laureate in Medicine 2015, and Relationships with the TCM Research Center Graz at the Medical University

\section{References}

1 Auszeichnung 2015: Medizin-Nobelpreis geht an drei Parasitenforscher. http://www.spiegel.de/wissenschaft/medizin/nobelpreis-medizin-2015-geht-an-william-c-campbell-satoshi-omura-youyoutu-a-1056187.html (accessed October 13, 2015).

2 Neill US: From branch to bedside: Youyou Tu is awarded the 2011 Lasker-DeBakey Clinical Medical Research Award for discovering artemisinin as a treatment for malaria. J Clin Invest 2011;121:3768-3773.

3 Biber P: Die Entdeckung eines Wundermittels gegen Malaria. http://www.srf.ch/wissen/mensch/dieentdeckung-eines-wundermittels-gegen-malaria (accessed October 14, 2015).

4 Malaria, RKI-Ratgeber für Ärzte. Robert Koch Institut. http://www.rki.de/DE/Content/Infekt/EpidBull/ Merkblaetter/Ratgeber_Malaria.html?nn=2374512\#doc2392924bodyText14 (accessed October 13, 2015).

5 Beifuss (Artemisia vulgaris). http://www.heilkraeuter.de/lexikon/beifuss.htm (accessed October 14, 2015).

6 Litscher G, Zhang WB, Yi SH, Wang L, Huang T, Gaischek I, Tian YY, Wang GJ: The future of acupuncture moxibustion: a transcontinental three-center pilot study using high-tech methods. Med Acupunct 2009;21:115121.

7 Zhao BX, Litscher G, Li J, Wang L, Cui YX, Huang C, Liu P: Effects of moxa (Artemisia vulgaris) smoke inhalation on heart rate and its variability. Chin Med 2011;2:53-57.

8 Litscher G: Bioengineering assessment of acupuncture, part 8: innovative moxibustion. Crit Rev Biomed Eng 2010;38:117-126.

9 Hageneder E: Moxibustion - Aktuelle naturwissenschaftliche Betrachtungen. Diploma thesis. Medical University of Graz, 2010, p 77.

10 Wang W: TCM products find their niche overseas. China Daily Business, 2015. 